Gut, 1979, 20, 1078-1082

\title{
Enterohepatic circulation rates of cholic acid and chenodeoxycholic acid in man
}

\author{
K. A. EINARSSON, ${ }^{1}$ S. M. GRUNDY, AND W. G. M. HARDISON \\ From the Department of Medicine, School of Medicine, University of California, San Diego, and \\ Veterans Administration Hospital, San Diego, California, USA
}

SUMMARY The rate of enterohepatic cycling of cholic acid and chenodeoxycholic acid was determined in five male subjects. Pool sizes were measured by isotope dilution technique after intraduodenal administration of ${ }^{14} \mathrm{C}$-labelled cholic and chenodeoxycholic acid. The hourly hepatic secretion rate of bile acids was determined by an intestinal perfusion technique. From these data the cycling frequency was calculated. Chenodeoxycholic acid circulated on an average 1.34 (range, 1.13-1.57) times faster than cholic acid, probably because chenodeoxycholic acid to a larger extent than cholic acid is absorbed from the proximal small intestine and thus partly bypasses the hepaticoileal circuit. This difference in cycling rate may have methodological as well as physiological implications.

Several studies have shown that biliary lipid composition is influenced by the types of bile acids in the enterohepatic circulation. Saturation of bile with cholesterol is decreased when bile contains predominantly chenodeoxycholic acid (CDCA) and is increased when cholic acid, and its derivative deoxycholic acid, are the major bile acids. Therefore, differences in the metabolism of these bile acids could be important in the regulation of the lipid composition of bile.

Several differences in the kinetics of cholic acid and CDCA have already been observed. It has been reported, for example, that the fractional turnover rate of cholic acid in the enterohepatic circulation exceeds that of CDCA. Another finding is that, after a meal, CDCA and deoxycholic acid show an earlier and more prolonged rise in serum concentrations compared with cholic acid (Angelin and Björkhem, 1977; Pennington et al., 1978; Ponz de Leon et al., 1978). A possible explanation for this difference in response could be that hepatic clearance of dihydroxy bile acids is less efficient than for cholic acid. However, another possibility is that after a meal there is a more rapid influx of dihydroxy acids from the intestine due to passive absorption from the jejunum. If this 'short-circuiting' of CDCA and deoxycholic acid is of quantitative importance, it should mean ${ }^{1}$ Address for reprint requests: Kurt A. Einarsson, MD, Department
of Medicine, Serafimerlasarettet, S-112 83 Stockholm, Sweden.

Received for publication 8 October 1979 that these bile acids circulate through the enterohepatic circulation at a greater rate than cholic acid.

To examine this possibility, pool sizes and secretion rates of cholic acid and CDCA were determined in five subjects, and from these data the cycling frequency of each was calculated. Our results show that CDCA circulates at a higher rate than cholic acid.

\section{Methods}

\section{SUBJECTS}

Five white men volunteered for this study, which was carried out on the Special Diagnostic and Treatment Unit of the Veterans Administration Hospital, San Diego, California. Clinical data are given in Table 1 . All subjects had normal bowel function and clinically intact enterohepatic circulation. The gallbladder was shown to be free from stones either by abdominal echography or by oral cholecystography. One patient had mild hypertriglyceridaemia and two were obese. At the time of study, all patients had normal routine tests of liver function. None of the subjects was taking hypolipidaemic or antibiotic drugs.

Subjects 1 to 4 were maintained at a constant weight with an ad libitum diet of solid type, containing about $40 \%$ of the calories as fat. Subject no. 5 was in the hospital primarily for weight loss taking a $960 \mathrm{kcal}$ diet of a liquid formula (Sustacal, Mead Johnson, Evansville, IN). 
Table 1 Clinical data

\begin{tabular}{|c|c|c|c|c|c|c|c|}
\hline \multirow{2}{*}{\multicolumn{2}{|c|}{ Subject }} & \multirow[b]{2}{*}{$\begin{array}{l}\text { Age } \\
(y r)\end{array}$} & \multirow[b]{2}{*}{$\begin{array}{l}\text { Weight } \\
(\mathrm{kg})\end{array}$} & \multirow[b]{2}{*}{$\begin{array}{l}\text { Height } \\
(\mathrm{cm})\end{array}$} & \multicolumn{2}{|c|}{ Plasma lipids } & \multirow[b]{2}{*}{ Clinical history } \\
\hline & & & & & $\begin{array}{l}\text { Cholesterol } \\
(\mathrm{mg} / \mathrm{dl})\end{array}$ & $\begin{array}{l}\text { Triglycerides } \\
(m g / d l)\end{array}$ & \\
\hline $\begin{array}{l}1 \\
2 \\
3 \\
4 \\
5\end{array}$ & $\begin{array}{l}\text { G.S. } \\
\text { T.P. } \\
\text { D.T. } \\
\text { J.H. } \\
\text { W.C. }\end{array}$ & $\begin{array}{l}59 \\
49 \\
49 \\
54 \\
48\end{array}$ & $\begin{array}{r}73 \\
110 \\
63 \\
79 \\
95\end{array}$ & $\begin{array}{l}179 \\
182 \\
173 \\
174 \\
172\end{array}$ & $\begin{array}{l}211 \\
157 \\
156 \\
190 \\
183\end{array}$ & $\begin{array}{l}215 \\
174 \\
118 \\
129 \\
158\end{array}$ & $\begin{array}{l}\overline{\text { Hypertension }} \\
\text { Myocardial infarction } \\
\overline{\text { Myocardial infarction, }} \\
\text { hypertension }\end{array}$ \\
\hline
\end{tabular}

HEPATIC SECRETION RATES OF BILE ACIDS

Outputs of biliary lipids were measured by the method of Grundy and Metzger (1972). In the evening before study, the subject swallowed a 3lumen tube which the next morning was positioned by radiography in the duodenum with two proximal outlets at the level of the ampulla of Vater and the other outlet $10 \mathrm{~cm}$ distally just past the ligament of Treitz. Liquid formula was then infused continuously through the most proximal lumen to provide the daily caloric requirement and to contract the gallbladder. Simultaneously, a small quantity of betasitosterol was infused as a marker. After an equilibration period of four hours, the duodenal contents were continuously aspirated in hourly $10 \mathrm{ml}$ aliquots from the second proximal and distal lumens for the next eight hours. Outputs of cholesterol into the duodenum were determined from the sample drawn at the distal outlet by use of the marker dilution principle. Bile salt secretion was then determined from the ratio of bile salt to cholesterol in the proximal samples. Total concentrations of bile acids were determined enzymatically. The coefficient of variation for hourly outputs of bile acids was, on average, $19 \%$.

For secretion rates of the individual bile acids, the composition of bile acids at the proximal site was determined. Bile salts were deconjugated by saponification for three hours in $1.25 \mathrm{M} \mathrm{NaOH}$ (at $121 \mathrm{C}^{\circ}$ $\left(250^{\circ} \mathrm{F}\right)$ and $\left.15 \mathrm{psi}\right)$. The deconjugated bile acids were extracted with ethyl ether after acidification, methylated, and analysed as trimethylsilyl ethers by gas-liquid chromatography using $1 \% \mathrm{Hi}$-Eff $8 \mathrm{BP}$ as the stationary phase. Corrections were made for different detector responses of the individual bile acids.

Daily secretion rates of bile acids were obtained by multiplying hourly secretion rates by 24 . This method has recently been validated when it was shown that hourly secretion $\times 24$ closely approximates secretion rates for a 24-hour period, as measured in patients given three formula meals a day (von Bergmann et al., 1977).

Ratios between glycine and taurine conjugated bile acids ( $\mathrm{G} / \mathrm{T}$ ratio) were determined in the proximal samples. The conjugated bile acids were separated by TLC. The plate was developed in isopentyl acetate-propionic acid-n-propanol-water (30:30:20: 15), dried and stained with iodine vapour. The gel containing the bands with glycine and taurine conjugated bile acids was scraped off separately and eluted with methanol. Bile acid concentration was measured by enzymatic method and the $G / T$ ratio was calculated.

\section{POOL SIZES OF BILE ACIDS}

These were measured simultaneously with hepatic secretion rates as described recently (Grundy, 1975). $\left[24-{ }^{14} \mathrm{C}\right]$ cholic acid $(5 \mu \mathrm{Ci})$ and $\left[24-{ }^{14} \mathrm{C}\right]$ chenodeoxycholic acid ( $5 \mu \mathrm{Ci}$ ) (New England Nuclear, Boston, Mass), dissolved in $10 \mathrm{ml}$ ethanol, were flushed through the distal outlet with water at the beginning of formula infusion. After four hours of equilibration with the endogenous pool of bile acids the specific radioactivity was determined for cholic acid and CDCA on hourly samples over the next eight hours. After deconjugation, the methyl esters of the bile acids were separated by thin layer chromatography using isooctane-ethyl acetate-acetic acid (10:10:2) as solvent. The bile acid bands were identified with iodine vapour. The gel containing the bands with cholic acid and CDCA was scraped off separately and eluted with methanol. One aliquot of the eluate was assayed for radioactivity. Another aliquot was evaporated, converted to the trimethylsilyl ether, and analysed by gas-liquid chromatography on $1 \%$ Hi-Eff 8 BP column. The specific radioactivity was calculated as cpm per $\mathrm{mg}$ of bile acid.

The bile acid pool size was calculated as radioactivity injected/average value of bile acid specific activity over the collecting period. This measurement of the bile acid pool size was based on the following assumptions: (1) that no significant amount of isotope was lost before complete mixing had occurred; (2) that the isotope was completely mixed with the endogenous bile acid; (3) that new synthesis of bile acid during the collection period was small compared 


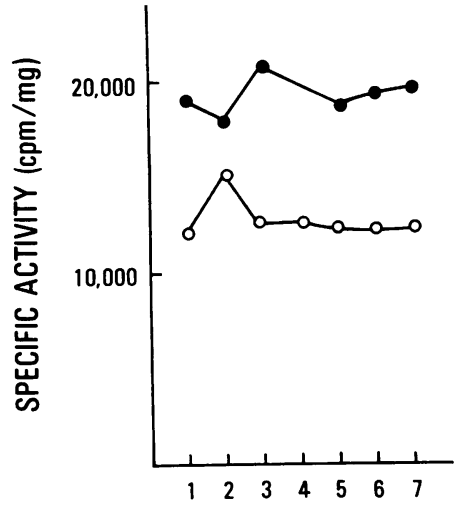

SAMPLE NUMBER

Figure Specific radioactivity of cholic acid (-) and chenodeoxycholic acid $(\mathrm{O}-\mathrm{O})$ in subject no. 1 (G.S.). ${ }^{14} \mathrm{C}$-labelled cholic acid and chenodeoxycholic acid were administered intraduodenally four hours before sample 1 .

with the pool size of the bile acid. The relative constancy of the specific activities of cholic acid as well as of CDCA during the sampling period, the coefficients of variation being 14 and $10 \%$, respectively, suggests that complete equilibration had occurred. A typical example is shown in the Figure. In two subjects the specific radioactivity of both bile acids declined towards the end of the period and therefore the two last values were excluded for these patients. Otherwise there was no consistent trend to a decline of the specific radioactivity indicating that the quantity of bile acid synthesised during the sampling period was negligible compared with the endogenous pool. It should be emphasised, however, that the pool size values obtained by the present technique represent the size of the circulating pool of bile acids. If some bile acids were sequestered in the gallbladder and did not enter the enterohepatic circulation during the study they would not be included in the measurement of pool size. Therefore the value for the pool size by the present method does not necessarily correspond exactly with that derived from the method of Lindstedt (1957).

\section{RECYCLING RATES OF BILE ACID POOLS}

These were obtained by dividing daily bile acid secretion by the pool size.

\section{Results}

The pattern of individual bile acids in duodenal bile is shown in Table 2. CDCA was the major bile acid in all five patients, comprising $46.7 \pm 2.0 \%$ of the total bile acids. The proportions of cholic acid and deoxycholic acid were $22.7 \pm 2.8$ and $25.8 \pm 2.4 \%$ respectively. Ursodeoxycholic acid and lithocholic acid were present in only small amounts.

The daily hepatic secretion of CDCA averaged $10764 \pm 1795 \mathrm{mg}$ and that of cholic acid $4833 \pm 316$ (Table 3). The pool size of CDCA exceeded that of cholic acid in all five subjects, the mean values being $1215 \pm 210$ and $714 \pm 96 \mathrm{mg}$, respectively. The ratios between the secretion rates of CDCA and cholic acid were higher than corresponding ratios between the

Table 2 Bile acid composition (molar percent) and $G / T$ ratio in duodenal bile of subjects

\begin{tabular}{|c|c|c|c|c|c|c|}
\hline Subject & Cholic & Chenodeoxycholic & Deoxycholic & Ursodeoxycholic & Lithocholic & $G / T$ \\
\hline $\begin{array}{l}1 \\
2 \\
3 \\
4 \\
5 \\
\text { Mean } \pm \\
\text { SEM }\end{array}$ & $\begin{array}{c}19 \cdot 2 \\
20 \cdot 2 \\
15 \cdot 8 \\
26 \cdot 3 \\
31 \cdot 8 \\
22 \cdot 7 \pm \\
2 \cdot 8\end{array}$ & $\begin{array}{c}44.4 \\
51 \cdot 6 \\
46.4 \\
50 \cdot 4 \\
40 \cdot 7 \\
46.7 \pm \\
2.0\end{array}$ & $\begin{array}{c}29 \cdot 2 \\
25 \cdot 1 \\
31 \cdot 8 \\
17 \cdot 8 \\
24 \cdot 9 \\
25 \cdot 8 \pm \\
2 \cdot 4\end{array}$ & $\begin{array}{l}6 \cdot 3 \\
2 \cdot 1 \\
2 \cdot 2 \\
2 \cdot 7 \\
0 \cdot 6 \\
2 \cdot 8 \pm \\
1 \cdot 0\end{array}$ & $\begin{array}{l}0 \cdot 9 \\
1 \cdot 5 \\
3 \cdot 7 \\
2 \cdot 8 \\
2 \cdot 0 \\
2 \cdot 2 \pm \\
0 \cdot 5\end{array}$ & $\begin{array}{l}1.95 \\
1.94 \\
2.91 \\
4.84 \\
1.81 \\
2.69 \pm \\
0.57\end{array}$ \\
\hline
\end{tabular}

Table 3 Daily secretion, pool size, and recycling rate of cholic acid and chenodeoxycholic acid in subjects

\begin{tabular}{|c|c|c|c|c|c|c|}
\hline \multirow[t]{2}{*}{ Subject } & \multicolumn{2}{|c|}{ Daily secretion (mg/day) } & \multicolumn{2}{|c|}{ Pool size (mg) } & \multicolumn{2}{|c|}{ Recycling rate (day-1) } \\
\hline & Cholic & Chenodeoxycholic & Cholic & Chenodeoxycholic & Cholic & Chenodeoxycholic \\
\hline
\end{tabular}


pool sizes of the two bile acids, which resulted in a higher cycling frequency of CDCA compared with that of cholic acid. Thus CDCA cycled on the average 1.34 times faster than cholic acid (range $1 \cdot 13-1 \cdot 57)$.

The $G / T$ ratio was within normal limits in all subjects (Table 2) and appeared to reflect neither the daily secretion nor the recycling rate of the bile acids.

\section{Discussion}

Previous studies have shown that the fractional turnover rate of CDCA is lower than that of cholic acid because of a better intestinal conservation. Thus, for given synthesis rates of the two bile acids, the pool size is larger for CDCA than for cholic acid. In most reports, cholic acid and CDCA are synthesised in a ratio of about $1 \cdot 5-2 \cdot 0: 1$, and ratios between the pool sizes are usually $1 \cdot 0-1 \cdot 5: 1$. For some unexplained reason, the CA:CDCA ratio in the present study was consistently below $1 \cdot 0$. Low ratios have been described for patients with cirrhosis of the liver and with hypercholesterolaemia, but our subjects showed no evidence of either disorder.

The better intestinal conservation of CDCA compared with that of cholic acid may be the result of a more efficient absorption of the former bile acid. Studies in rats have shown that less polar bile acidsin particular, glycine conjugated dihydroxy bile acids-can be absorbed by passive diffusion along the whole length of the small intestine; whereas the more polar bile acids, particularly the taurine conjugated trihydroxy bile acids, are absorbed only by active transport from the distal ileum (Dietschy, 1968; Schiff et al., 1972). These findings have been confirmed also for man. Direct, as well as indirect, measurements of bile acid absorption have shown that dihydroxy bile acids, preferentially glycine conjugated, are absorbed from jejunum (Hislop et al., 1967; Krag and Phillips, 1974; Angelin et al., 1976; Hardison, von Bergmann and Grundy, unpublished). All types of bile acids can be absorbed from the terminal ileum, but the active transport is more rapid for trihydroxy than for dihydroxy bile acids (Krag and Phillips, 1974). Proximal absorption of bile acids may well be an important component of the normal enterohepatic circulation in man, as $50-75 \%$ of the bile acids are conjugated with glycine and only 25 $50 \%$ with taurine. The early postprandial rise in serum dihydroxy bile acids has been attributed at least in part to rapid proximal reabsorption of these less polar bile acids (Angelin and Björkhem, 1977; Pennington et al., 1978; Ponz de Leon et al., 1978).

It has earlier been proposed that an enhanced cycling frequency of bile acids diminishes the pool because a certain percentage of the pool is lost with each circuit and, furthermore, a high cycling rate means a high flux of bile acids through the liver which suppresses synthesis (Low-Beer and Pomare, 1973; Northfield and Hofmann, 1973; Mok et al., 1977). Indeed, in most reports CDCA has a lower synthesis rate and a smaller pool size than cholic acid; for some reason, this was not the case in the present study. In spite of enhanced cycling frequency, CDCA has a lower fractional turnover rate than cholic acid, which may depend on a more complete absorption from the intestine due to the uptake from the proximal intestine. Furthermore, the portion of CDCA which is absorbed proximal to this site escapes $7 \alpha$-dehydroxylation in the colon, which may also contribute to the better conservation and the lower fractional turnover of CDCA compared with cholic acid.

The jejunal absorption of biie acids may be of great importance in patients with defective ileal absorption of bile acids. The ratio of glycine-taurine conjugated bile acids is greatly increased after ileectomy, and it has been suggested that this predominance of glycine conjugat $\mathrm{d}$ bile acids is partly due to the more efficient jejunal absorption of glycine conjugated than taurine conjugated bile acids with selective loss of the latter (Dowling, 1972).

The proximal absorption of bile acids may also be of importance during bile acid feeding, as the reserve of the ileal transport system seems to be small especially for dihydroxy bile acids (Krag and Phillips, 1974). The G/T ratio increases during bile acid feeding because of the limited supply of taurine but probably also because of an enhanced conservation of glycine conjugated bile acids.

Finally, the different cycling rates of cholic acid and CDCA may also have consequences for the method of determining total bile acid pool size described by Grundy (1975). In Table 4, we have calculated the total bile acid pool for the subjects, assuming that deoxycholic acid, ursodeoxycholic acid, and lithocholic acid circulated at the same rate

Table 4 Total bile acid pool in subjects calculated (I) according to Grundy (1975), (II) by technique used in present study assuming that all dihydroxy bile acids and lithocholic acid circulate at same rate in EHC, and (III) using Grundy's method and including correction factor for different cycling rates of $C A$ versus other bile acids

\begin{tabular}{llll}
\hline Subject & \multicolumn{3}{l}{ Total bile acid pool $(\mathrm{mg})$} \\
\cline { 2 - 4 } & $I$ & $I I$ & $I I I$ \\
\hline 1 & 3875 & 3024 & 3030 \\
2 & 5435 & 4121 & 4392 \\
3 & 4202 & 2909 & 3320 \\
4 & 2152 & 1979 & 1768 \\
5 & 1893 & 1735 & 1543 \\
Mean \pm SEM & $3511 \pm 662$ & $2754 \pm 425$ & $2811 \pm 525$ \\
\hline
\end{tabular}


as CDCA, which is reasonable for at least the two former bile acids as they are of about the same polarity as CDCA. The pool sizes of these bile acids were calculated from their ratios to CDCA in bile and the mean total bile acid pool was determined to be $2754 \pm 425 \mathrm{mg}$. This pool size value was compared with that obtained with the method described by Grundy (1975). Grundy's method assumes that all bile acids circulate at the same rate and only one labelled bile acid, $\left[{ }^{14} \mathrm{C}\right] \mathrm{CA}$, is administered to the patient. Considering our present data, that method would have given us a total pool size of $3511 \pm 662$ $\mathrm{mg}$ - that is, an overestimation of about $25 \%$. After correction for different cycling rates by multiplying the fraction of mono- and dihydroxy bile acids by 0.744 , which is the ratio between the cycling rates of cholic acid and CDCA, we obtained a total bile acid pool of $2811 \pm 525$, which is very close to the actual one (Table 4). Thus, if only labelled cholic acid is used, then accuracy is increased by using the correction factor. However, it should be pointed out that an accurate measurement of the total bile acid pool size by the technique used in this study requires determination of the pool sizes of the individual bile acids.

The authors wish to express their appreciation to Marjorie Whelan and Joan Rupp, and others of the nursing and dietetic services on the Special Diagnostic and Treatment Unit, Veterans Administration Hospital, San Diego, California, for their assistance on this project. Excellent technical assistance was provided by Janna Naylor, James Hobza, Robert Ronimus, and Lynne Lesh. This project was supported by the Medical Research Service of the Veterans Administration and by NIH Research Grants No. HL-14197, awarded by National Heart, Lung, and Blood Institute and No. AM-16667 from the National Institute of Arthritis, Metabolism, and Digestive Diseases PHS/DHEW. This investigation was also supported in part by a Public Health Service International Research Fellowship (No. 1 F05 TW02467-01).

\section{References}

Angelin, B., and Björkhem, I. (1977). Postprandial serum bile acids in healthy man. Evidence for differences in absorptive pattern between individual bile acids. Gut, 18, 606-609.

Angelin, B., Einarsson, K., and Hellström, K. (1976). Evidence for the absorption of bile acids in the proximal small intestine for normo- and hyperlipidemic subjects. Gut, 17, 420-425.

Dietschy, J. M. (1968). Mechanisms for the intestinal absorption of bile acids. Journal of Lipid Research, 9, 297-309.

Dowling, R. H. (1972). The enterohepatic circulation. Gastroenterology, 62, 122-140.

Grundy, S. M. (1975). Effects of polyunsaturated fats on lipid metabolism in patients with hypertriglyceridemia. Journal of Clinical Investigation, 55, 269-282.

Grundy, S. M., and Metzger, A. L. (1972). A physiologic method for estimation of hepatic secretion of biliary lipids in man. Gastroenterology, 62, 1200-1217.

Hislop, I. G., Hofmann, A. F., and Schoenfield, L. J. (1967). Determinants of the rate and site of bile acid absorption in man (Abstract). Journal of Clinical Investigation, 46, 1070-1071.

Krag, E., and Phillips, S. F. (1974). Active and passive bile acid absorption in man. Perfusion studies of the ileum and jejunum. Journal of Clinical Investigation, 53, 1686-1694.

Lindstedt, S. (1957). Turnover of cholic acid in man. Acta Physiologica Scandinavica, 40, 1-9.

Low-Beer, T. C., and Pomare, E. W. (1973). Regulation of bile salt pool size in man. British Medical Journal, 2, 338-340.

Mok, H. Y. I., von Bergmann, K., and Grundy, S. M(1977). Regulation of pool size of bile acids in man. Gastroenterology, 73, 684-690.

Northfield, T. C., and Hofmann, A. F. (1973). Biliary lipid secretion in gallstone patients. Lancet, 1, 747-748.

Pennington, C. R., Ross, P. E., and Bouchier, I. A. (1978). Fasting and postprandial serum bile acid concentrations in normal persons using an improved GLC method. Digestion, 17, 56-62.

Ponz de Leon, M., Murphy, G. M., and Dowling, R. H. (1978). Physiological factors influencing serum bile acid levels. Gut, 19, 32-39.

Schiff, E. R., Small, N. C., and Dietschy, J. M. (1972). Characterization of the kinetics of the passive and active transport mechanisms for bile acid absorption in the small intestine and colon of the rat. Journal of Clinical Investigation, 51, 1351-1362.

von Bergmann, K., Mok, H. Y. I., and Grundy, S. M. (1977). Effects of intermittent and continuous feeding on biliary lipid secretion in man. In Bile Acid Metabolism in Health and Disease, pp. 191-196. Edited by G. Paumgartner and A. Stiehl. MTP Press: Lancaster. 